\begin{tabular}{|c|c|}
\hline 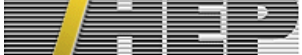 & PUBLISHED FOR SISSA BY SPRINGER \\
\hline & $\begin{array}{r}\text { ReCEIVED: June 9, } 2019 \\
\text { AcCEPted: June 12, } 2019 \\
\text { Published: June 19, } 2019\end{array}$ \\
\hline
\end{tabular}

\title{
Integral of two-loop modular graph functions ${ }^{1}$
}

\section{Eric D'Hoker}

Mani L. Bhaumik Institute for Theoretical Physics,

Department of Physics and Astronomy, University of California,

Los Angeles, CA 90095, U.S.A.

E-mail: dhoker@physics.ucla.edu

ABSTRACT: The integral of an arbitrary two-loop modular graph function over the fundamental domain for $\mathrm{SL}(2, \mathbb{Z})$ in the upper half plane is evaluated using recent results on the Poincaré series for these functions.

Keywords: Scattering Amplitudes, Superstrings and Heterotic Strings

ARXIV EPRINT: 1905.06217

\footnotetext{
${ }^{1}$ Research supported in part by the National Science Foundation under research grant PHY-16-19926.
} 


\section{Contents}

1 Introduction 1

2 Modular graph functions $\quad 2$

2.1 One-loop modular graph functions: Eisenstein series 4

2.2 Two-loop modular graph functions 4

3 Integrals of modular graph functions $\quad 5$

3.1 Integrals of Eisenstein series and their products 6

$\begin{array}{lll}3.2 & \text { Integral of two-loop modular graph functions of odd weight } & 7\end{array}$

3.3 Integral of two-loop modular graph functions of even weight 9

$\begin{array}{lll}\text { 3.3.1 Subtraction of the term linear in } \tau_{2} & 10\end{array}$

3.3.2 Unfolding the integral of the Poincaré series of $\hat{\mathcal{C}}_{u, v ; w} \quad 11$

$\begin{array}{lll}\text { 3.3.3 Carrying out the integral over } \tau_{2} & 12\end{array}$

$\begin{array}{ll}\text { 3.3.4 Evaluating } \mathcal{G}_{u, v ; w} & 13\end{array}$

4 Higher loops: open problems $\quad 14$

\section{Introduction}

A modular graph function is an $\mathrm{SL}(2, \mathbb{Z})$-invariant function on the Poincaré upper half plane $\mathcal{H}$ which is associated with a certain type of graph [1,2]. Modular graph functions provide a natural generalization to higher loop graphs of real-analytic Eisenstein series, which are associated with one-loop graphs. They are key building blocks for the integrand on the moduli space of genus-one Riemann surfaces of the low energy expansion of superstring theory to genus-one order $[3,4]$.

Modular graph functions obey a wealth of differential and algebraic identities [1, 5-10]; their Poincaré series was obtained for low weight in $[1,11,12]$ and for general weight in [13]; the Laurent polynomial part of their constant Fourier mode was derived for low weight in [1, 14] and for arbitrary weight at two-loop order in [15]; the full Fourier series at two-loop order was calculated in [13]. The significance of multiple zeta-values [16] in string theory and modular graph functions was explored in [14, 17-20], while relations with period integrals, mixed motives, and equivariant iterated Eisenstein integrals were studied in $[20-22]$.

String perturbation theory requires, however, not just the integrand but the actual integral of modular graph functions on the moduli space of genus-one Riemann surfaces $\mathcal{M}=\mathcal{H} / \mathrm{SL}(2, \mathbb{Z})$. The purpose of this paper is to define and evaluate such integrals for all modular graph functions associated with two-loop graphs. 
For modular functions which tend to zero at the cusp (cuspidal functions), such integrals may be computed using the Rankin-Selberg method, in terms of the constant Fourier mode of the cuspidal function [23, 24]. Modular graph functions, however, have polynomial growth at the cusp, just as real-analytic Eisenstein series do, and their integration requires regularization. A standard regularization is obtained by removing from the domain of integration $\mathcal{M}$ a small neighborhood of the cusp, and integrating over $\mathcal{M}_{L}$ defined for $L>1$ by,

$$
\mathcal{M}_{L}=\mathcal{M} \cap\{\operatorname{Im}(\tau)<L\} \quad \mathcal{M}=\left\{\tau \in \mathcal{H},|\tau| \geq 1,|\operatorname{Re}(\tau)| \leq \frac{1}{2}\right\}
$$

A generalization of the Rankin-Selberg method, which is applicable to modular functions with polynomial growth at the cusp, was introduced in [25] and evaluates integrals over $\mathcal{M}_{L}$ of expressions linear, bilinear, and trilinear in Eisenstein series.

The integrals over moduli space required for superstring amplitudes are unique and free of divergences, but their construction requires subtle analytic continuation in the kinematic variables of the amplitude. The existence of this analytic continuation for the four-graviton amplitude at genus one was proven in [26], where the results of the analytic continuation were used to obtain the mass-shifts and decay widths of massive string states. The evaluation of the amplitude to low orders in the kinematic parameters was carried out in $[1,3,4]$. The integrals of modular graph functions regularized by integrating over the truncated fundamental domain $\mathcal{M}_{L}$ provide a key part of the evaluation of the analytically continued amplitudes. Recent investigations of the transcendentality properties of the genus-one amplitude in [27] directly motivate the problems addressed in the present paper.

The organization of the remainder of this paper is as follows. In section 2 we shall briefly review the definition and basic properties of modular graph functions in terms of their Kronecker-Eisenstein and Poincaré series representations. The main results of the paper are presented in Theorems 3.1 and 3.2 of section 3, respectively giving the (regularized) integrals for two-loop modular graphs functions of arbitrary odd and even weight. We conclude in section 4 with a discussion of open problems and speculations regarding the integrals of higher loop modular graph functions.

\section{Modular graph functions}

In this section we shall give a brief review of the definition and basic properties of modular graph functions needed in the sequel of the paper.

A decorated connected graph $(\Gamma, A, B)$ with $V$ vertices and $R$ edges is specified by the connectivity matrix $\Gamma$ of the graph and the decoration $(A, B)$ of the edges of the graph. The components of the connectivity matrix $\Gamma$ are denoted by $\Gamma_{v r}$ where $v=1, \cdots, V$ labels the vertices and $r=1, \cdots, R$ labels the edges. No edge is allowed to begin and end on the same vertex: when edge $r$ contains vertex $v$ we have $\Gamma_{v r}= \pm 1$ while otherwise $\Gamma_{v r}=0$. The decoration $(A, B)$ of the edges is specified by two arrays of exponents $a_{r}, b_{r}$,

$$
A=\left[a_{1}, \cdots, a_{R}\right] \quad B=\left[b_{1}, \cdots, b_{R}\right]
$$


where $a_{r}, b_{r} \in \mathbb{C}$ with $a_{r}-b_{r} \in \mathbb{Z}$ for all $r=1, \cdots, R$. To a connected decorated graph $(\Gamma, A, B)$ we associate a complex-valued function on $\mathcal{H}$, defined by a multiple KroneckerEisenstein sum over $p_{1}, \cdots, p_{R}$ in $\Lambda^{\prime}=\Lambda \backslash\{0\}$ where $\Lambda=\mathbb{Z}+\tau \mathbb{Z}$ for $\tau \in \mathcal{H}$,

$$
\mathcal{C}_{\Gamma}\left[\begin{array}{l}
A \\
B
\end{array}\right](\tau)=\sum_{p_{1}, \ldots, p_{R} \in \Lambda^{\prime}} \prod_{v=1}^{V} \delta\left(\sum_{s=1}^{R} \Gamma_{v s} p_{s}\right) \prod_{r=1}^{R} \frac{\tau_{2}^{\frac{1}{2} a_{r}+\frac{1}{2} b_{r}}}{\pi^{\frac{1}{2} a_{r}+\frac{1}{2} b_{r}}\left(p_{r}\right)^{a_{r}}\left(\bar{p}_{r}\right)^{b_{r}}}
$$

The Kronecker $\delta$ equals 1 when its argument vanishes and 0 otherwise. Absolute convergence of the sums in (2.2) is ensured by a system of inequalities on the combinations $\operatorname{Re}\left(a_{r}+b_{r}\right)$, beyond which the functions of (2.2) may be defined by analytic continuation in $a_{r}+b_{r}$.

The function $\mathcal{C}_{\Gamma}$ defined on $\mathcal{H}$ in (2.2) vanishes whenever $\Gamma$ becomes disconnected upon severing a single edge, and factorizes whenever $\Gamma$ is the union of two subgraphs whose intersection consists of a single vertex. Furthermore, $\mathcal{C}_{\Gamma}$ is invariant under the action of $\operatorname{SL}(2, \mathbb{Z})$ on $\tau$ if and only if the sums of the $A$ and $B$ exponents are equal to one another,

$$
w=a_{1}+\cdots+a_{R}=b_{1}+\cdots+b_{R}
$$

The number $w$ is the weight, while $R-V+1$ is the number of loops of the modular graph function $\mathcal{C}_{\Gamma}$. Bivalent vertices may be eliminated by suitably increasing the values of the exponents $a_{r}, b_{r}$. Henceforth, we shall assume that $\Gamma$ remains connected upon removing any single edge or vertex and that the equality of the sums of $A$ and $B$ exponents in (2.3) holds.

It was shown in [2] that the behavior near the cusp of an arbitrary modular graph function of weight $w \geq 2$, whose exponents satisfy (2.3), is governed by a Laurent polynomial in $\tau_{2}$ of degree $(w, 1-w)$ up to exponentially suppressed terms,

$$
\mathcal{C}_{\Gamma}\left[\begin{array}{l}
A \\
B
\end{array}\right](\tau)=\sum_{k=1-w}^{w} \mathcal{L}_{k}\left(4 \pi \tau_{2}\right)^{k}+\mathcal{O}\left(e^{-2 \pi \tau_{2}}\right)
$$

where $\mathcal{L}_{k}$ are constants. Its Poincaré series may be obtained by the arguments used in [13] for the more restricted class of dihedral modular graph functions, namely for graphs containing two vertices whose valence is larger than 2. The Poincaré series of an arbitrary modular graph function of weight $w$ with respect to the coset $\Gamma_{\infty} \backslash \operatorname{PSL}(2, \mathbb{Z})$ is given by,

$$
\mathcal{C}_{\Gamma}\left[\begin{array}{l}
A \\
B
\end{array}\right](\tau)=\sum_{g \in \Gamma_{\infty} \backslash \operatorname{PSL}(2, \mathbb{Z})} \Lambda_{\Gamma}\left[\begin{array}{l}
A \\
B
\end{array}\right](g \tau)
$$

where $\Gamma_{\infty}$ is the Borel subgroup of translations of $\tau$, and the seed function $\Lambda_{\Gamma}$ is given by,

$$
\Lambda_{\Gamma}\left[\begin{array}{l}
A \\
B
\end{array}\right](\tau)=\frac{\tau_{2}^{w}}{\pi^{w}} \sum_{n \neq 0} \sum_{p_{1}, \ldots, p_{R} \in \Lambda^{\prime}} \delta\left(p_{R}-n\right) \prod_{v=1}^{V} \delta\left(\sum_{s=1}^{R} \Gamma_{v s} p_{s}\right) \prod_{r=1}^{R} \frac{1}{\left(p_{r}\right)^{a_{r}}\left(\bar{p}_{r}\right)^{b_{r}}}
$$

Using the same methods as were used in [13] for two-loop modular graph functions, one shows that the Poincare series in the general case is absolutely convergent whenever the original Kronecker-Eisenstein series is absolutely convergent. We shall next provide more explicit formulas in the cases of one-loop and two-loop modular graph functions. 


\subsection{One-loop modular graph functions: Eisenstein series}

A connected one-loop modular graph function of weight $w$ reduces to an Eisenstein series,

$$
E_{w}(\tau)=\sum_{p \in \Lambda^{\prime}} \frac{\tau_{2}^{w}}{\pi^{w}|p|^{2 w}}
$$

which satisfies the differential equation,

$$
\Delta E_{w}=w(w-1) E_{w} \quad \Delta=4 \tau_{2}^{2} \partial_{\bar{\tau}} \partial_{\tau}
$$

The Fourier series representation of $E_{w}$ in the variable $\tau_{1}$ is given by,

$$
E_{w}(\tau)=c_{w} \tau_{2}^{w}+\tilde{c}_{w} \tau_{2}^{1-w}+\frac{8 \tau_{2}^{\frac{1}{2}}}{\Gamma(w)} \sum_{N=1}^{\infty} N^{w-\frac{1}{2}} \sigma_{1-2 w}(N) K_{w-\frac{1}{2}}\left(2 \pi N \tau_{2}\right) \cos \left(2 \pi N \tau_{1}\right)
$$

Here, $K_{s}$ is the modified Bessel function, and we have used the following notations,

$$
c_{w}=\frac{2 \zeta(2 w)}{\pi^{w}} \quad \tilde{c}_{w}=\frac{2 \Gamma\left(w-\frac{1}{2}\right) \zeta(2 w-1)}{\Gamma(w) \pi^{w-\frac{1}{2}}} \quad \sigma_{s}(N)=\sum_{0<d \mid N} d^{s}
$$

where $\zeta$ is the Riemann zeta-function. The Poincaré series of $E_{w}$,

$$
E_{w}(\tau)=\sum_{g \in \Gamma_{\infty} \backslash \operatorname{PSL}(2, \mathbb{Z})} c_{w}(\operatorname{Im} g \tau)^{w}
$$

is absolutely convergent throughout $\mathcal{H}$ for $\operatorname{Re}(w)>1$.

\subsection{Two-loop modular graph functions}

The Kronecker-Eisenstein sum for a two-loop modular graph function with non-negative integer exponents $a_{r}, b_{r}$ and weight $w=a_{1}+a_{2}+a_{3}=b_{1}+b_{2}+b_{3}$ simplifies as follows,

$$
\mathcal{C}\left[\begin{array}{lll}
a_{1} & a_{2} & a_{3} \\
b_{1} & b_{2} & b_{3}
\end{array}\right](\tau)=\sum_{p_{1}, p_{2}, p_{3} \in \Lambda^{\prime}} \frac{\tau_{2}^{w} \delta_{p_{1}+p_{2}+p_{3}, 0}}{\pi^{w} p_{1}^{a_{1}} p_{2}^{a_{2}} p_{3}^{a_{3}} \bar{p}_{1}^{b_{1}} \bar{p}_{2}^{b_{2}} \bar{p}_{3}^{b_{3}}}
$$

Every two-loop modular graph function admits a linear decomposition onto a subset of modular graph functions of a simplified type,

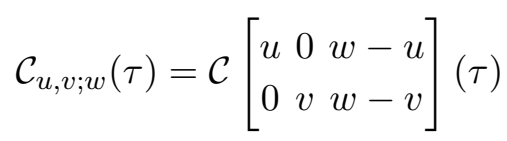

The decomposition was proven in Proposition 2.1 of [13] and is given by,

$$
\begin{aligned}
\mathcal{C}\left[\begin{array}{lll}
a_{1} & a_{2} & a_{3} \\
b_{1} & b_{2} & b_{3}
\end{array}\right]= & \sum_{u=1}^{a_{1}} \sum_{v=w-b_{3}}^{w-1}(-)^{a_{3}+b_{2}+u+v+w}\left(\begin{array}{c}
w-u-1-a_{3} \\
a_{2}-1
\end{array}\right)\left(\begin{array}{c}
v-1-b_{2} \\
b_{1}-1
\end{array}\right) \mathcal{C}_{u, v ; w}+(1 \leftrightarrow 2) \\
& +\sum_{u=w-a_{1}}^{w-1} \sum_{v=w-b_{1}}^{w-1}(-)^{a_{3}+b_{2}+u+v}\left(\begin{array}{c}
u-1-a_{3} \\
a_{2}-1
\end{array}\right)\left(\begin{array}{c}
v-1-b_{2} \\
b_{3}-1
\end{array}\right) \mathcal{C}_{u, v ; w}+(1 \leftrightarrow 2)
\end{aligned}
$$


where the terms $(1 \leftrightarrow 2)$ are obtained by swapping the pairs $\left(a_{1}, b_{1}\right)$ with $\left(a_{2}, b_{2}\right)$ leaving $\left(a_{3}, b_{3}\right)$ unchanged. Therefore, the Fourier series, Poincaré series, and integral of an arbitrary two-loop modular graph function may be expressed in terms of the functions $\mathcal{C}_{u, v ; w}$.

The Fourier series representation of $\mathcal{C}_{u, v ; w}$ in the variable $\tau_{1}$ was obtained explicitly in [13]. The Laurent polynomial part of the constant Fourier modes is given as follows,

$$
\mathcal{C}_{u, v ; w}(\tau)=\ell_{w}\left(4 \pi \tau_{2}\right)^{w}+\sum_{k=0}^{w-2} \ell_{w-2 k-3} \frac{\zeta(2 k+3)}{\left(4 \pi \tau_{2}\right)^{-w+2 k+3}}+\frac{\ell_{2-w}}{\left(4 \pi \tau_{2}\right)^{w-2}}+\mathcal{O}\left(e^{-4 \pi \tau_{2}}\right)
$$

where $\ell_{w}, \ell_{w-2 k-3} \in \mathbb{Q}$, the coefficients $\ell_{w-2 k-3}$ are given by,

$$
\begin{aligned}
\ell_{w-2 k-3}= & \frac{(-)^{k} \zeta(2 w-2 k-4)}{(2 \pi)^{2 w-2 k-4}}\left(4(-)^{v+w}\left(\begin{array}{c}
2 k+2 \\
w-u-1
\end{array}\right)\left(\begin{array}{c}
u+v-2 w+2 k+3 \\
u-2 w+2 k+4
\end{array}\right)\right. \\
& \left.+2\left(\begin{array}{c}
2 k+2 \\
-u-v+2 k+4
\end{array}\right)\left(\begin{array}{c}
u+v-2 \\
v-1
\end{array}\right)\right)+(u \leftrightarrow v)
\end{aligned}
$$

and $\ell_{2-w}$ is a bilinear in odd zeta-values with rational coefficients. The explicit expressions for $\ell_{w}$ and $\ell_{2-w}$, the non-constant Fourier modes, and the exponentially suppressed part of the constant Fourier mode were also obtained in [13] but will not be needed here.

The Poincaré series of $\mathcal{C}_{u, v ; w}$ with respect to the coset $\Gamma_{\infty} \backslash \operatorname{PSL}(2, \mathbb{Z})$ is given by,

$$
\mathcal{C}_{u, v ; w}(\tau)=\sum_{g \in \Gamma_{\infty} \backslash \operatorname{PSL}(2, \mathbb{Z})} \Lambda_{u, v ; w}(g \tau)
$$

The seed function $\Lambda_{u, v ; w}$ was computed in [13] and is given by,

$$
\Lambda_{u, v ; w}(\tau)=\ell_{w}\left(4 \pi \tau_{2}\right)^{w}+\sum_{m, n \neq 0} \sum_{\mu \in \mathbb{Z}} \frac{(-)^{v} \tau_{2}^{w}}{\pi^{w} n^{2 w-u-v}(m \tau+\mu)^{u}(m \bar{\tau}+\mu+n)^{v}}
$$

The expressions for the seed function $\Lambda_{u, v ; w}(\tau)$ in terms of elementary functions, given in [13], will not be needed here.

\section{Integrals of modular graph functions}

The purpose of this paper is to integrate modular graph functions over $\mathcal{M}_{L}$ for finite $L>1$,

$$
\mathcal{S}_{\Gamma}\left[\begin{array}{l}
A \\
B
\end{array}\right](L)=\int_{\mathcal{M}_{L}} \frac{d^{2} \tau}{\tau_{2}^{2}} \mathcal{C}_{\Gamma}\left[\begin{array}{l}
A \\
B
\end{array}\right](\tau)
$$

The $L$-dependence of $\mathcal{S}_{\Gamma}$ is given by the integral of the constant Fourier mode of $\mathcal{C}_{\Gamma}$,

$$
\mathcal{S}_{\Gamma}\left[\begin{array}{l}
A \\
B
\end{array}\right](L)=\mathcal{S}_{\Gamma}\left[\begin{array}{l}
A \\
B
\end{array}\right](1)+\int_{1}^{L} \frac{d \tau_{2}}{\tau_{2}^{2}} \int_{0}^{1} d \tau_{1} \mathcal{C}_{\Gamma}\left[\begin{array}{l}
A \\
B
\end{array}\right](\tau)
$$

thereby determining the full integral $\mathcal{S}_{\Gamma}$ in terms of the constant Fourier mode up to an additive constant. As $L \rightarrow \infty$, the behavior of $\mathcal{S}_{\Gamma}$ is determined by the Laurent polynomial 
part of the constant Fourier mode, up to this additive integration constant and up to exponentially suppressed corrections,

$$
\mathcal{S}_{\Gamma}(L)=\mathcal{L}_{c}+\mathcal{L}_{1} \ln L+\sum_{\substack{k=1-w \\ k \neq 1}}^{w} \frac{\mathcal{L}_{k}}{k-1} L^{k-1}+\mathcal{O}\left(e^{-4 \pi L}\right)
$$

For string theory applications it is the constant $\mathcal{L}_{c}$, which is not determined by the Laurent polynomial, in which we are most interested. In the sequel we shall begin by reviewing the results of Zagier for the case when the modular function is linear or bilinear in the Eisenstein series. Next, we shall present new results for the case where $\mathcal{C}_{\Gamma}$ is an arbitrary two-loop modular graph function, and obtain the integrals using the Poincaré series of $\mathcal{C}_{\Gamma}$.

\subsection{Integrals of Eisenstein series and their products}

The integral of $E_{w}$ over the domain $\mathcal{M}_{L}$ may be simplified by using the differential equation (2.8) and Green's theorem to recast it as an integral over the boundary of $\mathcal{M}_{L}$,

$$
w(w-1) \int_{\mathcal{M}_{L}} \frac{d^{2} \tau}{\tau_{2}^{2}} E_{w}=\int_{\mathcal{M}_{L}} \frac{d^{2} \tau}{\tau_{2}^{2}} \Delta E_{w}=\left.\int_{0}^{1} d \tau_{1} \partial_{\tau_{2}} E_{w}\right|_{\tau_{2}=L}
$$

Using the Fourier series for $E_{w}$ of (2.9) in the right most integral, we see that only the constant Fourier mode contributes and the integral may be evaluated exactly,

$$
\int_{\mathcal{M}_{L}} \frac{d^{2} \tau}{\tau_{2}^{2}} E_{w}=\frac{c_{w} L^{w-1}}{w-1}-\frac{\tilde{c}_{w} L^{-w}}{w}
$$

Away from $w=0$ and $w=1$ the integral is an analytic function of $w$. For $0<\operatorname{Re}(w)<1$, the right side tends to zero as $L \rightarrow \infty$, resulting in the vanishing of the integral of $E_{w}$ over the full fundamental domain $\mathcal{M},{ }^{1}$

$$
\int_{\mathcal{M}} \frac{d^{2} \tau}{\tau_{2}^{2}} E_{w}=\lim _{L \rightarrow \infty} \int_{\mathcal{M}_{L}} \frac{d^{2} \tau}{\tau_{2}^{2}} E_{w}=0
$$

The integral of a product of two Eisenstein series may be simplified in a similar manner,

$$
\begin{aligned}
\left(w^{\prime}\left(w^{\prime}-1\right)-w(w-1)\right) \int_{\mathcal{M}_{L}} \frac{d^{2} \tau}{\tau_{2}^{2}} E_{w} E_{w^{\prime}} & =\int_{\mathcal{M}_{L}} \frac{d^{2} \tau}{\tau_{2}^{2}}\left(E_{w} \Delta E_{w^{\prime}}-E_{w^{\prime}} \Delta E_{w}\right) \\
& =\left.\int_{0}^{1} d \tau_{1}\left(E_{w} \partial_{\tau_{2}} E_{w^{\prime}}-E_{w^{\prime}} \partial_{\tau_{2}} E_{w}\right)\right|_{\tau_{2}=L}
\end{aligned}
$$

In this case the non-constant Fourier modes of $E_{w}$ and $E_{w^{\prime}}$ will contribute to the boundary term as well, but their effect will be exponentially suppressed for large $L$, and we obtain an equation which is equivalent to the Maass-Selberg relation [25],

$$
\int_{\mathcal{M}_{L}} \frac{d^{2} \tau}{\tau_{2}^{2}} E_{w} E_{w^{\prime}}=\frac{c_{w} c_{w^{\prime}} L^{w+w^{\prime}-1}}{w+w^{\prime}-1}+\frac{\tilde{c}_{w} \tilde{c}_{w^{\prime}} L^{1-w-w^{\prime}}}{1-w-w^{\prime}}+\frac{c_{w} \tilde{c}_{w^{\prime}} L^{w-w^{\prime}}}{w-w^{\prime}}+\frac{\tilde{c}_{w} c_{w^{\prime}} L^{w^{\prime}-w}}{w^{\prime}-w}+\mathcal{O}\left(e^{-4 \pi L}\right)
$$

\footnotetext{
${ }^{1}$ It has been assumed in [25] that analytic continuation in $w$ may be used to set the integral over $\mathcal{M}$ to zero throughout $w \in \mathbb{C}$, but we shall not need or use this assumption here.
} 
In this case the region in $w, w^{\prime}$ where the right side tends to zero as $L \rightarrow \infty$ is empty. However, if $\operatorname{Re}\left(w-w^{\prime}\right)>0$ and $\operatorname{Re}\left(w+w^{\prime}\right)>1$ we may subtract a suitably chosen linear combination of $E_{w+w^{\prime}}$ and $E_{w-w^{\prime}+1}$ to cancel the growing terms in the limit $L \rightarrow \infty$, leading to the following convergent integral [25],

$$
\int_{\mathcal{M}} \frac{d^{2} \tau}{\tau_{2}^{2}}\left(E_{w} E_{w^{\prime}}-\frac{c_{w} c_{w^{\prime}}}{c_{w+w^{\prime}}} E_{w+w^{\prime}}-\frac{c_{w} \tilde{c}_{w^{\prime}}}{c_{w-w^{\prime}+1}} E_{w-w^{\prime}+1}\right)=0
$$

The integral of $E_{w}^{2}$ may be obtained by taking the limit as $w^{\prime} \rightarrow w$ of (3.8) and we find,

$$
\lim _{L \rightarrow \infty}\left(\int_{\mathcal{M}_{L}} \frac{d^{2} \tau}{\tau_{2}^{2}}\left\{E_{w}^{2}-\frac{c_{w}^{2}}{c_{2 w}} E_{2 w}\right\}-2 c_{w} \tilde{c}_{w} \ln L\right)=\tilde{c}_{w} \frac{\partial c_{w}}{\partial w}-c_{w} \frac{\partial \tilde{c}_{w}}{\partial w}
$$

This result was obtained in appendix A of [4].

\subsection{Integral of two-loop modular graph functions of odd weight}

When the weight $w \geq 3$ of the modular graph function $\mathcal{C}_{u, v ; w}$ is odd, the terms in (2.15) that grow with $\tau_{2}$ near the cusp are the leading term proportional to $\tau_{2}^{w}$, as well as the sub-leading terms with $0 \leq 2 k \leq w-5$. Therefore, the integral of $\mathcal{C}_{u, v ; w}$ over $\mathcal{M}$ diverges near the cusp. We shall define a convergent integral by subtracting from $\mathcal{C}_{u, v ; w}$ a linear combination of Eisenstein series $E_{s}$ for integer with $2 \leq s \leq w$, chosen so as to cancel the growing behavior near the cusp. Specifically, we define the following modular function,

$$
\hat{\mathcal{C}}_{u, v ; w}=\mathcal{C}_{u, v ; w}-\ell_{w} \frac{(2 \pi)^{2 w}}{2 \zeta(2 w)} E_{w}-\sum_{k=0}^{\frac{w-5}{2}} \ell_{w-2 k-3} \frac{(2 \pi)^{2 w-4 k-6} \zeta(2 k+3)}{2 \zeta(2 w-4 k-6)} E_{w-2 k-3}
$$

For the range of $k$ in the above sum, the expression for the coefficients $\ell_{w-2 k-3}$ given in (2.16) simplifies, since $0 \leq 2 k \leq w-5$ and $u<w$ imply $u-2 w+2 k+4 \leq u-w-1<0$ and, as a result, the first term in (2.16) for $\ell_{w-2 k-3}$ vanishes. The remaining term is manifestly symmetric under $u \leftrightarrow v$ and may be expressed as follows,

$$
\ell_{w-2 k-3}=4(-)^{k}\left(\begin{array}{c}
2 k+2 \\
u+v-2
\end{array}\right)\left(\begin{array}{c}
u+v-2 \\
u-1
\end{array}\right) \frac{\zeta(2 w-2 k-4)}{(2 \pi)^{2 w-2 k-4}}
$$

where the first binomial coefficient vanishes for $2 k+4<u+v$. By construction, the function $\hat{\mathcal{C}}_{u, v ; w}$ tends to a constant at the cusp, and is integrable on the full fundamental domain $\mathcal{M}$. The integral is given by the following theorem.

Theorem 3.1 For $w=2 \kappa+1 \geq 3$ an odd integer and positive integers $u, v$ satisfying $u+v \geq 3$ and $u, v \leq w-1$, the integral of $\hat{\mathcal{C}}_{u, v ; w}$ over $\mathcal{M}$,

$$
\hat{\mathcal{S}}_{u, v ; w}=\int_{\mathcal{M}} \frac{d^{2} \tau}{\tau_{2}^{2}} \hat{\mathcal{C}}_{u, v ; w}(\tau)
$$

evaluates to,

$$
\hat{\mathcal{S}}_{u, v ; w}=2 \pi \zeta(2 \kappa+1)\left(\begin{array}{c}
2 \kappa-1 \\
2 \kappa+1-u-v
\end{array}\right)\left(\begin{array}{c}
u+v-2 \\
u-1
\end{array}\right) \frac{B_{2 \kappa}}{(2 \kappa) !}
$$


where $B_{2 \kappa}$ are the Bernoulli numbers. The integral of $\mathcal{C}_{u, v ; w}$ over $\mathcal{M}_{L}$ follows by combining (3.5) and (3.11), up to contributions which vanish for $L \rightarrow \infty$.

Note that the first binomial coefficient vanishes when $w<u+v$, and that $\hat{\mathcal{S}}_{u, v ; w}$ is given by a rational number times an odd zeta-value, and is therefore a single-valued zeta-value.

To prove the theorem, namely equation (3.14), we evaluate the integral of $\hat{\mathcal{C}}_{u, v ; w}$ using the Poincaré series for $\hat{\mathcal{C}}_{u, v ; w}$, which may be obtained by combining the Poincaré series of $\mathcal{C}_{u, v ; w}$ and $E_{s}$ using (3.11). The result is the following Poincare series, which is absolutely convergent [13],

$$
\hat{\mathcal{C}}_{u, v ; w}(\tau)=\sum_{g \in \Gamma_{\infty} \backslash \operatorname{PSL}(2, \mathbb{Z})} \hat{\Lambda}_{u, v ; w}(g \tau)
$$

The seed function $\hat{\Lambda}_{u, v ; w}$ is obtained by combining the seed function of $\mathcal{C}_{u, v ; w}$ in (2.18) with the seed function for $E_{s}$ in (2.11) using again (3.11), and we have,

$$
\begin{aligned}
\hat{\Lambda}_{u, v ; w}(\tau)= & \sum_{m, n \neq 0} \sum_{\mu \in \mathbb{Z}} \frac{(-)^{v} \tau_{2}^{w}}{\pi^{w} n^{2 w-u-v}(m \tau+\mu)^{u}(m \bar{\tau}+\mu+n)^{v}} \\
& -\sum_{k=0}^{\frac{w-5}{2}} 4(-)^{k} \zeta(2 k+3) \zeta(2 w-2 k-4)\left(\begin{array}{c}
2 k+2 \\
-u-v+2 k+4
\end{array}\right)\left(\begin{array}{c}
u+v-2 \\
v-1
\end{array}\right) \frac{\left(2 \tau_{2}\right)^{w-2 k-3}}{(2 \pi)^{w-1}}
\end{aligned}
$$

Using the standard unfolding trick for the integral of a Poincaré series, we have,

$$
\hat{\mathcal{S}}_{u, v ; w}=\int_{0}^{\infty} \frac{d \tau_{2}}{\tau_{2}^{2}} \int_{0}^{1} d \tau_{1} \hat{\Lambda}_{u, v ; w}(\tau)
$$

The sum over the integer $\mu$ in the first line may be carried out by partial fraction decomposition and use of the following standard summation formula,

$$
\sum_{\mu \in \mathbb{Z}} \frac{1}{(z+\mu)^{k+1}}=i \pi \frac{(-)^{k}}{k !} \frac{d^{k}}{d z^{k}}\left(\frac{1+e^{2 \pi i z}}{1-e^{2 \pi i z}}\right)
$$

The integral over $\tau_{1}$ of the partial fractions projects onto the constant Fourier mode, which contributes only for $k=0$, and we obtain,

$$
\int_{0}^{1} d \tau_{1} \sum_{\mu \in \mathbb{Z}} \frac{1}{(m \tau+\mu)^{u}(m \bar{\tau}+\mu+n)^{v}}=-2 \pi i \frac{(-)^{v} \varepsilon(m)\left(\begin{array}{c}
u+v-2 \\
u-1
\end{array}\right)}{\left(2 i m \tau_{2}-n\right)^{u+v-1}}
$$

where $\varepsilon(m)$ evaluates to 1 for $m>0,-1$ for $m<0$, and 0 for $m=0$. As a result, we find,

$$
\begin{aligned}
\hat{\mathcal{S}}_{u, v ; w}= & \frac{\left(\begin{array}{c}
u+v-2 \\
u-1
\end{array}\right)}{\pi^{w-1}} \int_{0}^{\infty} d \tau_{2}\left[\sum_{m, n=1}^{\infty} \frac{\tau_{2}^{w-2}}{n^{2 w-u-v}}\left(\frac{-4 i}{\left(2 i m \tau_{2}-n\right)^{u+v-1}}-\frac{-4 i}{\left(-2 i m \tau_{2}-n\right)^{u+v-1}}\right)\right. \\
& \left.-\sum_{k=0}^{\frac{w-5}{2}} 4(-)^{k} \zeta(2 k+3) \zeta(2 w-2 k-4)\left(\begin{array}{c}
2 k+2 \\
-u-v+2 k+4
\end{array}\right) \frac{\tau_{2}^{w-2 k-5}}{2^{2 k+2}}\right]
\end{aligned}
$$

To obtain the first term we have used the invariance under $(m, n) \rightarrow(-m,-n)$ to restrict the sum over $m$ to $m>0$ upon including a factor of 2 , and then decomposed the $n$-sum into 
its contributions from positive and negative $n$. The presence of the subtraction terms on the second line above, which resulted from the subtraction of Eisenstein series, guarantees the absolute convergence of the integral.

One would like to rescale $\tau_{2}$ in the first line of the integrand to factor out the $m$ and $n$-dependences. But the integral over each line separately does not converge, so we need to rescale all terms in the integrand simultaneously. To do so, we substitute for $\zeta(2 k+3)$ and $\zeta(2 w-2 k-4)$ their infinite series representations respectively in the summation variables $m$ and $n$, and then change variables from $\tau_{2}$ to $x=2 m \tau_{2} / n$. Doing so, all dependence on $m$ and $n$ factors out and may be summed in terms of Riemann zeta-values. Next, we use the fact that, for $w$ odd, the integrand is even in $x$ in order to extend the integration range to all of $\mathbb{R}$, upon including a factor of $\frac{1}{2}$, and we obtain,

$$
\begin{aligned}
\hat{\mathcal{S}}_{u, v ; w}= & \frac{\left(\begin{array}{c}
u+v-2 \\
u-1
\end{array}\right) \zeta(w-1) \zeta(w)}{(2 \pi)^{w-1}} \int_{-\infty}^{\infty} d x\left[\frac{-2 i x^{w-2}}{(i x-1)^{u+v-1}}+\frac{2 i x^{w-2}}{(-i x-1)^{u+v-1}}\right. \\
& \left.-\sum_{k=0}^{\frac{w-5}{2}} 4(-)^{k}\left(\begin{array}{c}
2 k+2 \\
-u-v+2 k+4
\end{array}\right) x^{w-2 k-5}\right]
\end{aligned}
$$

The subtraction terms guarantee that the integrand tends to zero as $1 / x^{2}$ for large $x$, so we may close the contour of integration in the upper half plane and evaluate the integral via the residue of the integrand at the single pole at $x=i$. Finally, the even zeta-values may be converted into Bernoulli numbers using the relation,

$$
\zeta(2 \kappa)=\frac{1}{2}(-)^{\kappa+1}(2 \pi)^{2 \kappa} \frac{B_{2 \kappa}}{(2 \pi)^{2 \kappa}}
$$

which gives the result announced in (3.14) of Theorem 3.1, thereby completing its proof.

We note that the value of the integral $\hat{\mathcal{S}}_{u, v ; w}$ is closely related to the term of order $\tau_{2}^{0}$ in the Laurent polynomial of $\mathcal{C}_{u, v ; w}$, which is given by $\ell_{0} \zeta(w)$. This term corresponds to $2 k=w-3$ in the sum of (2.15) and, since we have $u-2 w+2 k+4=u-w+1<0$, the first term in the general expression for $\ell_{w-2 k-3}$ does not contribute. As a result, we find,

$$
\hat{\mathcal{S}}_{u, v ; w}=\pi \frac{w-u-v+1}{w-1} \ell_{0} \zeta(w)
$$

where we recall that $\ell_{0}$ is a rational number dependent on $u, v, w$.

\subsection{Integral of two-loop modular graph functions of even weight}

The case of even $w$ presents a complication, which was absent for odd $w$, due to the presence in the Laurent polynomial of $\mathcal{C}_{u, v ; w}$ of the term linear in $\tau_{2}$. On the one hand this term will lead to a logarithmic divergence of the integral of $\mathcal{C}_{u, v ; w}$ at the cusp. On the other hand, it cannot be regularized by subtracting an Eisenstein series since $E_{s}$ has a pole in $s$ at $s=1$. We shall be led to subtracting a more complicated modular graph functions whose integral can be evaluated with the use of the differential equation it satisfies, along the lines of the integrals carried out in subsection 3.1 for products of Eisenstein series. The result is given by the following theorem. 
Theorem 3.2 For $w=2 \kappa \geq 4$ an even integer and positive integers $u$, satisfying $u+v \geq 3$ and $u, v \leq w-1$, we define the modular function $\tilde{\mathcal{C}}_{u, v ; w}$ in terms of $\mathcal{C}_{u, v ; w}$ as follows,

$$
\tilde{\mathcal{C}}_{u, v ; w}=\mathcal{C}_{u, v ; w}-\ell_{w} \frac{(2 \pi)^{2 w} E_{w}}{2 \zeta(2 w)}-\sum_{k=0}^{\frac{w-6}{2}} \ell_{w-2 k-3} \frac{(2 \pi)^{2 w-4 k-6} \zeta(2 k+3)}{2 \zeta(2 w-4 k-6)} E_{w-2 k-3}
$$

The coefficients $\ell_{w-2 k-3}$ were given in (3.12) for the range $0 \leq 2 k \leq w-6$ needed here. Its regularized integral, which is defined by,

$$
\tilde{\mathcal{S}}_{u, v ; w}=\lim _{L \rightarrow \infty}\left(\int_{\mathcal{M}_{L}} \frac{d^{2} \tau}{\tau_{2}^{2}} \tilde{\mathcal{C}}_{u, v ; w}(\tau)-4 \pi \ell_{1} \zeta(w-1) \ln (2 L)\right)
$$

evaluates as follows,

$$
\begin{aligned}
\tilde{\mathcal{S}}_{u, v ; w}= & \pi \zeta(2 \kappa-1)(-)^{\kappa+1} \frac{B_{2 \kappa}}{(2 \kappa) !}\left(\begin{array}{c}
u+v-2 \\
u-1
\end{array}\right) \mathcal{G}_{u, v ; w} \\
& +4 \pi \ell_{1} \zeta(2 \kappa-1)\left(\frac{\zeta^{\prime}(2 \kappa)}{\zeta(2 \kappa)}-\frac{\zeta^{\prime}(2 \kappa-1)}{\zeta(2 \kappa-1)}\right)
\end{aligned}
$$

The function $\mathcal{G}_{u, v ; w}$ takes rational values and may be expressed as a linear combination of finite harmonic sums with integer coefficients whose detailed form will be given in (3.45).

Before proving the theorem, a comment on the transcendentality weight of the result is in order. The first term in $\hat{\mathcal{S}}_{u, v ; w}$ is a rational number times $\pi \zeta(2 \kappa-1)$ times $\mathcal{G}_{u, v ; w}$, which is a finite sum with integer coefficients of finite harmonic sums. It is argued in [27] that such finite harmonic sums should be assigned transcendental weight one. Similarly, it is argued in [27] that the logarithmic derivatives that enter $\hat{\mathcal{S}}_{u, v ; w}$ should also be assigned transcendental weight one. Therefore, both terms in $\hat{\mathcal{S}}_{u, v ; w}$ have transcendental weight $w+1=2 \kappa+1$.

To prove Theorem 3.2 we first verify the existence of a finite limit in (3.25) by observing that the function $\tilde{\mathcal{C}}_{u, v ; w}$ grows as $4 \pi \tau_{2} \ell_{1} \zeta(2 \kappa-1)$ near the cusp, where $\ell_{1}$ is,

$$
\ell_{1}=-2\left(\begin{array}{c}
2 \kappa-2 \\
u+v-2
\end{array}\right)\left(\begin{array}{c}
u+v-2 \\
u-1
\end{array}\right) \frac{B_{2 \kappa}}{(2 \kappa) !}
$$

so that its integral over $\mathcal{M}_{L}$ grows as $4 \pi \ell_{1} \zeta(2 \kappa-1) \ln (L)$.

We cannot directly apply the unfolding trick to the computation of the integral of $\tilde{\mathcal{C}}_{u, v ; w}$ using its Poincaré series, since the simplest unfolding is valid for convergent integrals on the entire fundamental domain $\mathcal{M}$. To obtain a convergent integral we must make a further subtraction to eliminate the term linear in $\tau_{2}$. One option would be to subtract a term proportional to $E_{s}^{2}$ with $s=w / 2$. The problem with this option is that its Poincaré series with seed function proportional to $\tau_{2}^{s} E_{s}$ fails to be integrable due to a divergence as $\tau_{2} \rightarrow 0$.

\subsubsection{Subtraction of the term linear in $\tau_{2}$}

Instead we shall choose to subtract a term proportional to a fixed modular graph function,

$$
C_{2,1,1}-\frac{2}{3} E_{4}=\mathcal{C}_{2,2 ; 4}-\frac{3}{2}\left(\mathcal{C}_{2,3 ; 4}+\mathcal{C}_{3,2 ; 4}\right)-\frac{2}{3} E_{4}
$$


whose behavior at the cusp may be deduced from (2.15) and is given by,

$$
C_{2,1,1}-\frac{2}{3} E_{4}=\frac{\pi \zeta(3) \tau_{2}}{45}+\mathcal{O}\left(\tau_{2}^{-1}\right)
$$

Using this result, we define the modular function $\hat{\mathcal{C}}_{u, v ; w}$ by,

$$
\hat{\mathcal{C}}_{u, v ; w}=\tilde{\mathcal{C}}_{u, v ; w}-\frac{180}{\zeta(3)} \ell_{1} \zeta(w-1)\left(C_{2,1,1}-\frac{2}{3} E_{4}\right)
$$

By construction, $\hat{\mathcal{C}}_{u, v ; w}$ tends to zero at the cusp and is integrable on $\mathcal{M}$. Its integral,

$$
\hat{\mathcal{S}}_{u, v ; w}=\int_{\mathcal{M}} \frac{d^{2} \tau}{\tau_{2}^{2}} \hat{\mathcal{C}}_{u, v ; w}(\tau)
$$

may be simply related to the integral $\tilde{\mathcal{S}}_{u, v ; w}$ we seek by,

$$
\tilde{S}_{u, v ; w}=\hat{\mathcal{S}}_{u, v ; w}+4 \pi \ell_{1} \zeta(w-1) \lim _{L \rightarrow \infty}\left(\frac{45}{\pi \zeta(3)} \int_{\mathcal{M}_{L}} \frac{d^{2} \tau}{\tau_{2}^{2}}\left\{C_{2,1,1}-\frac{2}{3} E_{4}\right\}-\ln (2 L)\right)
$$

The contribution under the limit may be computed using the differential equation,

$$
(\Delta-2) C_{2,1,1}=9 E_{4}-E_{2}^{2}
$$

to express $C_{2,1,1}$ as a linear combination of $\Delta C_{2,1,1}, E_{4}$ and $E_{2}^{2}$,

$$
C_{2,1,1}-\frac{2}{3} E_{4}=\frac{1}{2} \Delta\left(C_{2,1,1}-\frac{2}{3} E_{4}\right)+\frac{1}{2}\left(E_{2}^{2}-\frac{7}{3} E_{4}\right)
$$

The integral of the first term on the right side may be evaluated using Green's theorem, while the integral of the second term may be evaluated using (3.10) for the case $w=w^{\prime}=2$. Putting all together, we have,

$$
\lim _{L \rightarrow \infty}\left(\frac{45}{\pi \zeta(3)} \int_{\mathcal{M}_{L}} \frac{d^{2} \tau}{\tau_{2}^{2}}\left\{C_{2,1,1}-\frac{2}{3} E_{4}\right\}-\ln (2 L)\right)=\frac{\zeta^{\prime}(4)}{\zeta(4)}-\frac{\zeta^{\prime}(3)}{\zeta(3)}
$$

\subsubsection{Unfolding the integral of the Poincaré series of $\hat{\mathcal{C}}_{u, v ; w}$}

It remains to evaluate the integral $\hat{S}_{u, v ; w}$, which we do using its Poincaré series and the standard unfolding trick on the fundamental domain $\mathcal{M}$. The Poincaré series is given by,

$$
\hat{\mathcal{C}}_{u, v ; w}(\tau)=\sum_{g \in \Gamma_{\infty} \backslash \operatorname{PSL}(2, \mathbb{Z})} \hat{\Lambda}_{u, v ; w}(g \tau)
$$

in terms of which the integral is given by,

$$
\hat{\mathcal{S}}_{u, v ; w}=\int_{0}^{\infty} \frac{d \tau_{2}}{\tau_{2}^{2}} \int_{0}^{1} d \tau_{1} \hat{\Lambda}_{u, v ; w}(\tau)
$$


The seed function is obtained from the seed function $\Lambda_{u, v ; w}$ in (2.18), the seed function $c_{w} \tau_{2}^{w}$ for $E_{w}$, and the seed function for $C_{2,1,1}$, expressed in terms of the seed functions for $\mathcal{C}_{2,2 ; 4}, \mathcal{C}_{2,3 ; 4}$ and $\mathcal{C}_{3,2 ; 4}$, and we have,

$$
\begin{aligned}
\hat{\Lambda}_{u, v ; w}(\tau)= & \sum_{m, n \neq 0} \sum_{\mu \in \mathbb{Z}} \frac{(-)^{v} \tau_{2}^{w} n^{u+v}}{\pi^{w} n^{2 w}(m \tau+\mu)^{u}(m \bar{\tau}+\mu+n)^{v}} \\
& -\sum_{k=0}^{\frac{w-6}{2}} 4(-)^{k} \zeta(2 k+3) \zeta(2 w-2 k-4)\left(\begin{array}{c}
2 k+2 \\
-u-v+2 k+4
\end{array}\right)\left(\begin{array}{c}
u+v-2 \\
v-1
\end{array}\right) \frac{\left(2 \tau_{2}\right)^{w-2 k-3}}{(2 \pi)^{w-1}} \\
& -\frac{180 \tau_{2}^{4}}{\zeta(3) \pi^{4}} \zeta(w-1) \ell_{1} \sum_{m, n \neq 0} \sum_{\mu \in \mathbb{Z}}\left[\frac{1}{n^{4}(m \tau+\mu)^{2}(m \bar{\tau}+\mu+n)^{2}}\right. \\
& +\frac{\frac{3}{2} \quad \frac{3}{2}}{n^{3}(m \tau+\mu)^{2}(m \bar{\tau}+\mu+n)^{3}}-\frac{n^{3}(m \tau+\mu)^{3}(m \bar{\tau}+\mu+n)^{2}}{n^{3}(m 8}
\end{aligned}
$$

The summation and integration procedures are analogous to those we used in the case of $w$ odd, and we shall be brief on the parts of the calculation that are similar. We carry out partial fraction decompositions in the variable $\mu$, using the general formula,

$$
\frac{1}{(\mu+x)^{u}(\mu+y)^{v}}=\sum_{k=1}^{u} \frac{(-)^{v}\left(\begin{array}{c}
u+v-k-1 \\
u-k
\end{array}\right)}{(\mu+x)^{k}(x-y)^{u+v-k}}+\sum_{k=1}^{v} \frac{(-)^{u}\left(\begin{array}{c}
u+v-k-1 \\
v-k
\end{array}\right)}{(\mu+y)^{k}(y-x)^{u+v-k}}
$$

and use (3.18) to perform the sums over $\mu$ and (3.19) to do the integrals over $\tau_{1}$. Next, we exploit the symmetry of the summand under $(m, n) \rightarrow(-m,-n)$ to restrict the sums to $m>0$ upon including a factor of 2 and separate the contributions from positive and negative $n$. Finally, we express $\zeta(2 k+3)$ and $\zeta(2 w-2 k-4)$ as sums respectively over variables $m$ and $n$ as we did in the case of odd $w$. The result of these manipulations is as follows,

$$
\begin{aligned}
\int_{0}^{1} d \tau_{1} \hat{\Lambda}_{u, v ; w}(\tau)= & \frac{\left(\begin{array}{c}
u+v-2 \\
u-1
\end{array}\right)}{(2 \pi)^{w-1}} \sum_{m, n=1}^{\infty}\left[\frac{\left(2 \tau_{2}\right)^{w}}{n^{2 w-u-v}}\left(\frac{-2 i}{\left(2 i m \tau_{2}-n\right)^{u+v-1}}+\frac{2 i}{\left(-2 i m \tau_{2}-n\right)^{u+v-1}}\right)\right. \\
& -\sum_{k=0}^{\frac{w-6}{2}} 4(-)^{k} \frac{1}{m^{2 k+3} n^{2 w-2 k-4}}\left(\begin{array}{c}
2 k+2 \\
-u-v+2 k+4
\end{array}\right)\left(2 \tau_{2}\right)^{w-2 k-3} \\
& +\frac{180 \tau_{2}^{4}}{\zeta(3) \pi^{4}} \zeta(w-1) \zeta(w)(-)^{\frac{w}{2}}\left(\begin{array}{c}
w-2 \\
w-u-v
\end{array}\right)\left(\frac{16 i}{n^{4}\left(2 i m \tau_{2}-n\right)^{3}}\right. \\
& \left.\left.+\frac{16 i}{n^{4}\left(2 i m \tau_{2}+n\right)^{3}}+\frac{72 i}{n^{3}\left(2 i m \tau_{2}-n\right)^{4}}-\frac{72 i}{n^{3}\left(2 i m \tau_{2}+n\right)^{4}}\right)\right]
\end{aligned}
$$

\subsubsection{Carrying out the integral over $\tau_{2}$}

In carrying out the remaining integral over $\tau_{2}$, a crucial subtlety arises which did not occur in the case of odd $w$. The partial fraction decomposition of the integrand now contains an infinite sub-series which decays to zero as $1 / \tau_{2}$ at the cusp. To interchange the summations in the infinite series and the integration, and to carry out the integrations term by term, 
we shall first regularize the integral by imposing a cutoff $\tau_{2}<L$, and treat the terms in $1 / \tau_{2}$ separately. To do so, we introduce the following notation,

$$
\hat{\mathcal{S}}_{u, v ; w}=\frac{\left(\begin{array}{c}
u+v-2 \\
u-1
\end{array}\right)}{(2 \pi)^{w-1}}\left(\mathcal{F}_{u, v ; w}^{+}+\mathcal{F}_{u, v ; w}^{-}\right)
$$

The contribution $\mathcal{F}_{u, v ; w}^{-}$collects all the terms of order $1 / \tau_{2}$ in the partial fraction decomposition of the integrand, and is given by,

$$
\begin{aligned}
\mathcal{F}_{u, v ; w}^{-}= & 4(-)^{\frac{w}{2}}\left(\begin{array}{c}
w-2 \\
w-u-v
\end{array}\right) \lim _{L \rightarrow \infty} \int_{0}^{L} d \tau_{2} \sum_{m, n=1}^{\infty}\left[\frac{1}{n^{w} m^{w-1}}\left(\frac{1}{\tau_{2}+\frac{i n}{m}}+\frac{1}{\tau_{2}-\frac{i n}{m}}\right)\right. \\
& \left.-\frac{\zeta(w-1) \zeta(w)}{\zeta(3) \zeta(4) n^{4} m^{3}}\left(\frac{1}{\tau_{2}+\frac{i n}{m}}+\frac{1}{\tau_{2}-\frac{i n}{m}}\right)\right]
\end{aligned}
$$

The integrals are readily evaluated. In the limit $L \rightarrow \infty$, all dependence on $L$ drops out of the summations over $m, n$. The summations over $m, n$ may be performed in terms of the Riemann zeta function and its first derivative, and we find,

$$
\mathcal{F}_{u, v ; w}^{-}=8(-)^{\frac{w}{2}}\left(\begin{array}{c}
w-2 \\
w-u-v
\end{array}\right) \zeta(w) \zeta(w-1)\left(\frac{\zeta^{\prime}(w)}{\zeta(w)}-\frac{\zeta^{\prime}(w-1)}{\zeta(w-1)}-\frac{\zeta^{\prime}(4)}{\zeta(4)}+\frac{\zeta^{\prime}(3)}{\zeta(3)}\right)
$$

The contribution $\mathcal{F}_{u, v ; w}^{+}$collects all the remaining terms in the integral. Changing variables from $\tau_{2}$ to $y=2 m \tau_{2} / n$ in this absolutely convergent integral, the summations over $m$ and $n$ may be performed in terms of Riemann zeta-values. The terms in $1 / y$ which remain after this change of variables cancel in the integrand, and we are left with,

$$
\mathcal{F}_{u, v ; w}^{+}=\zeta(w) \zeta(w-1) \mathcal{G}_{u, v ; w}
$$

where the function $\mathcal{G}_{u, v ; w}$ is given by the integral,

$$
\begin{aligned}
\mathcal{G}_{u, v ; w}= & \int_{0}^{\infty} d y\left(\frac{4(-i)^{u+v} y^{w-2}}{(y+i)^{u+v-1}}+\frac{4 i^{u+v} y^{w-2}}{(y-i)^{u+v-1}}-\sum_{k=0}^{\frac{w-6}{2}} 8(-)^{k}\left(\begin{array}{c}
2 k+2 \\
-u-v+2 k+4
\end{array}\right) y^{w-2 k-5}\right. \\
& \left.-(-)^{\frac{w}{2}}\left(\begin{array}{c}
w-2 \\
w-u-v
\end{array}\right)\left(\frac{4 y^{2}}{(y+i)^{3}}+\frac{4 y^{2}}{(y-i)^{3}}-\frac{18 i y^{2}}{(y+i)^{4}}+\frac{18 i y^{2}}{(y-i)^{4}}\right)\right)
\end{aligned}
$$

Assembling all contributions to $\tilde{\mathcal{S}}_{u, v ; w}$ we observe that the contributions involving $\zeta^{\prime}(3)$ and $\zeta^{\prime}(4)$ cancel out between $\mathcal{F}_{u, v ; w}^{-}$and the combination of (3.32) and (3.35), and we obtain the result announced in Theorem 3.2, with the expression for $\mathcal{G}_{u, v ; w}$ given by (3.45).

\subsubsection{Evaluating $\mathcal{G}_{u, v ; w}$}

Finally, we evaluate $\mathcal{G}_{u, v ; w}$ by decomposing the integrand into partial fractions. All polynomially growing or constant contributions cancel by construction. Similarly, all terms that grow as $1 / y$ for large $y$ also cancel one another. Integrating the remaining rational functions produces only rational numbers, and we find,

$$
\mathcal{G}_{u, v ; w}=24(-)^{\frac{w}{2}}\left(\begin{array}{c}
w-2 \\
u+v-2
\end{array}\right)+8(-)^{\frac{w}{2}} \sum_{\ell=1}^{u+v-2}\left(\begin{array}{c}
w-2 \\
u+v-2-\ell
\end{array}\right) \frac{(-)^{\ell}}{\ell}
$$


Defining harmonic sums by,

$$
H_{\ell}=\sum_{m=1}^{\ell} \frac{1}{m} \quad H_{0}=0
$$

the function $\mathcal{G}_{u, v ; w}$ becomes a sum, with integer coefficients, of harmonic sums,

$$
\mathcal{G}_{u, v ; w}=24(-)^{\frac{w}{2}}\left(\begin{array}{c}
w-2 \\
u+v-2
\end{array}\right) H_{1}+8(-)^{\frac{w}{2}} \sum_{\ell=1}^{u+v-2}(-)^{\ell}\left(\begin{array}{c}
w-2 \\
u+v-2-\ell
\end{array}\right)\left(H_{\ell}-H_{\ell-1}\right)
$$

\section{Higher loops: open problems}

The regularized integrals of various individual modular graph functions at three-loop order and beyond have been evaluated using a combination of techniques, sometimes specific to a given modular graph function under study. General techniques include reducing the loop order of the integrand by the use of the algebraic identities and exposing total derivatives by the use of the differential identities satisfied by the modular graph functions. Although a general algorithm is known to find all algebraic identities at a given weight [7,9], the procedure for finding the identities is involved, and has been systematically worked out only to weight six included, with some identities known also at weight seven [9]. A different, but related, algorithm based on iterated Eisenstein integrals has been developed in [20]. An explicit expression for the integral of a modular graph functions of arbitrary loop order and arbitrary weight has not, however, been obtained thus far.

It is possible, however, to formulate the algorithm by which the integral of a modular graph function of arbitrary loop order and weight can be evaluated using only elementary integrals. We shall now describe the steps required. Consider a modular graph function $\mathcal{C}_{\Gamma}$ of weight $w$ given by an absolutely convergent Kronecker-Eisenstein series in (2.2), its Laurent polynomial in (2.4), and its absolutely convergent Poincaré series in (2.6). Note that the values of $\mathcal{L}_{k}$ in the Laurent polynomial of (2.4) are not known for arbitrary loop order and weight, but can be (laboriously) calculated at any order if needed. Following the procedure used at two-loops, we construct a new modular function,

$$
\hat{\mathcal{C}}_{\Gamma}\left[\begin{array}{l}
A \\
B
\end{array}\right]=\mathcal{C}_{\Gamma}\left[\begin{array}{l}
A \\
B
\end{array}\right]-\sum_{k=2}^{w} \frac{(4 \pi)^{k} \mathcal{L}_{k}}{c_{k}} E_{k}-\frac{180 \mathcal{L}_{1}}{\zeta(3)}\left(C_{2,1,1}-\frac{2}{3} E_{4}\right)
$$

By construction, $\hat{\mathcal{C}}_{\Gamma}$ tends to a constant at the cusp and is therefore integrable on $\mathcal{M}$. The integral of $\hat{\mathcal{C}}_{\Gamma}$ is obtained using the unfolding trick, and may be expressed in terms of the integral of its Poincaré seed,

$$
\int_{\mathcal{M}} \frac{d^{2} \tau}{\tau_{2}^{2}} \hat{\mathcal{C}}_{\Gamma}\left[\begin{array}{l}
A \\
B
\end{array}\right](\tau)=\int_{0}^{\infty} \frac{d \tau_{2}}{\tau_{2}^{2}} \int_{0}^{1} d \tau_{1} \hat{\Lambda}_{\Gamma}\left[\begin{array}{l}
A \\
B
\end{array}\right](\tau)
$$


where the seed function $\hat{\Lambda}_{\Gamma}$ is given in terms of the seed function $\Lambda_{\Gamma}$ for $\mathcal{C}_{\Gamma}$ by,

$$
\begin{aligned}
\hat{\Lambda}_{\Gamma}\left[\begin{array}{l}
A \\
B
\end{array}\right](\tau)= & \Lambda_{\Gamma}\left[\begin{array}{l}
A \\
B
\end{array}\right](\tau)-\sum_{k=2}^{w} \mathcal{L}_{k}\left(4 \pi \tau_{2}\right)^{k} \\
& -\frac{90 \tau_{2}^{4} \mathcal{L}_{1}}{\zeta(3) \pi^{4}} \sum_{m, n \neq 0} \sum_{\mu \in \mathbb{Z}}\left[\frac{2}{n^{4}(m \tau+\mu)^{2}(m \bar{\tau}+\mu+n)^{2}}\right. \\
& \left.+\frac{3}{n^{3}(m \tau+\mu)^{2}(m \bar{\tau}+\mu+n)^{3}}-\frac{3}{n^{3}(m \tau+\mu)^{3}(m \bar{\tau}+\mu+n)^{2}}\right]
\end{aligned}
$$

By construction, the integral of the Poincaré seed $\hat{\Lambda}_{\Gamma}$ converges absolutely. The open problem is to obtain the constant Fourier mode of the seed function $\hat{\Lambda}_{\Gamma}$ for arbitrary loop order and weight. To any given order, this problem may be solved by partial fraction decomposition of the denominators, and use of the summation formula (3.18).

We conclude by offering a speculation on the structure of the integrals of modular graph functions, defined above, for arbitrary loop order and weight. It has been proven that the coefficients $\mathcal{L}_{k}$ of the Laurent polynomial of $\mathcal{C}_{\Gamma}$ are generated by multiple zeta-values with rational coefficients [5], and it was argued that they are in fact generated by single-valued multiple zeta-values $[5,14]$. We speculate that the integrals are generated by multiple zeta-values (times a trivial factor of $\pi$ due to the volume of the fundamental domain), and derivates with respect to the arguments of zeta-values and multiple zeta-values.

\section{Acknowledgments}

We are happy to thank Bill Duke, Michael Green, Justin Kaidi, and Pierre Vanhove for various collaborations which led to the present paper, and for subsequent discussions. Also, we are happy to acknowledge the Niels Bohr International Academy in Copenhagen for the warm hospitality extended to the author during part of this work. This research is supported in part by the National Science Foundation under research grant PHY-16-19926.

Open Access. This article is distributed under the terms of the Creative Commons Attribution License (CC-BY 4.0), which permits any use, distribution and reproduction in any medium, provided the original author(s) and source are credited.

\section{References}

[1] E. D'Hoker, M.B. Green and P. Vanhove, On the modular structure of the genus-one Type II superstring low energy expansion, JHEP 08 (2015) 041 [arXiv: 1502.06698] [INSPIRE].

[2] E. D’Hoker, M.B. Green, Ö. Gürdoğan and P. Vanhove, Modular Graph Functions, Commun. Num. Theor. Phys. 11 (2017) 165 [arXiv:1512.06779] [InSPIRE].

[3] M.B. Green and P. Vanhove, The Low-energy expansion of the one loop type-II superstring amplitude, Phys. Rev. D 61 (2000) 104011 [hep-th/9910056] [INSPIRE].

[4] M.B. Green, J.G. Russo and P. Vanhove, Low energy expansion of the four-particle genus-one amplitude in type-II superstring theory, JHEP 02 (2008) 020 [arXiv:0801.0322] [INSPIRE]. 
[5] E. D'Hoker, M.B. Green and P. Vanhove, Proof of a modular relation between 1-, 2- and 3-loop Feynman diagrams on a torus, arXiv:1509.00363 [INSPIRE].

[6] A. Basu, Poisson equation for the Mercedes diagram in string theory at genus one, Class. Quant. Grav. 33 (2016) 055005 [arXiv: 1511.07455] [INSPIRE].

[7] E. D'Hoker and M.B. Green, Identities between Modular Graph Forms, J. Number Theor. 189 (2018) 25 [arXiv:1603.00839] [INSPIRE].

[8] A. Basu, Proving relations between modular graph functions, Class. Quant. Grav. 33 (2016) 235011 [arXiv: 1606.07084] [INSPIRE].

[9] E. D'Hoker and J. Kaidi, Hierarchy of Modular Graph Identities, JHEP 11 (2016) 051 [arXiv: 1608.04393] [INSPIRE].

[10] A. Kleinschmidt and V. Verschinin, Tetrahedral modular graph functions, JHEP 09 (2017) 155 [arXiv: 1706. 01889] [INSPIRE].

[11] O. Ahlén and A. Kleinschmidt, $D^{6} R^{4}$ curvature corrections, modular graph functions and Poincaré series, JHEP 05 (2018) 194 [arXiv: 1803.10250] [INSPIRE].

[12] D. Dorigoni and A. Kleinschmidt, Modular graph functions and asymptotic expansions of Poincaré series, arXiv:1903.09250 [INSPIRE].

[13] E. D'Hoker and J. Kaidi, Modular graph functions and odd cuspidal functions. Fourier and Poincaré series, JHEP 04 (2019) 136 [arXiv: 1902.04180] [INSPIRE].

[14] F. Zerbini, Single-valued multiple zeta values in genus 1 superstring amplitudes, Commun. Num. Theor. Phys. 10 (2016) 703 [arXiv:1512.05689] [INSPIRE].

[15] E. D'Hoker and W. Duke, Fourier series of modular graph functions, arXiv:1708.07998 [INSPIRE].

[16] J. Blumlein, D.J. Broadhurst and J.A.M. Vermaseren, The Multiple Zeta Value Data Mine, Comput. Phys. Commun. 181 (2010) 582 [arXiv:0907.2557] [InSPIRE].

[17] O. Schlotterer and S. Stieberger, Motivic Multiple Zeta Values and Superstring Amplitudes, J. Phys. A 46 (2013) 475401 [arXiv:1205.1516] [InSPIRE].

[18] J. Broedel, O. Schlotterer and S. Stieberger, Polylogarithms, Multiple Zeta Values and Superstring Amplitudes, Fortsch. Phys. 61 (2013) 812 [arXiv:1304.7267] [INSPIRE].

[19] J. Broedel, C.R. Mafra, N. Matthes and O. Schlotterer, Elliptic multiple zeta values and one-loop superstring amplitudes, JHEP 07 (2015) 112 [arXiv:1412.5535] [INSPIRE].

[20] J. Broedel, O. Schlotterer and F. Zerbini, From elliptic multiple zeta values to modular graph functions: open and closed strings at one loop, JHEP 01 (2019) 155 [arXiv:1803.00527] [INSPIRE].

[21] F. Brown, A class of non-holomorphic modular forms I, arXiv:1707.01230 [INSPIRE].

[22] F. Brown, A class of non-holomorphic modular forms II, equivariant iterated Eisenstein integrals, arXiv:1708.03354.

[23] R. Rankin, Contributions to the theory of Ramanujan's function $\tau(n)$ and similar arithmetic functions. I, Math. Proc. Camb. Philos. Soc. 35 (1939) 351.

[24] A. Selberg, Bemerkungen über eine Dirichetsche Reihe, die mit der Theorie der Modulformen nahe verbunden ist, Arch. Math. Naturvid. 43 (1940) 47. 
[25] D. Zagier, The Rankin-Selberg method for automorphic functions which are not of rapid decay, J. Fac. Sci. Tokyo 28 (1982) 415.

[26] E. D'Hoker and D.H. Phong, The Box graph in superstring theory, Nucl. Phys. B 440 (1995) 24 [hep-th/9410152] [INSPIRE].

[27] E. D'Hoker and M.B. Green, Exploring transcendentality in superstring amplitudes, arXiv: 1906.01652 [INSPIRE]. 